\title{
RECURRENT INCREASED NUCHAL TRANSLUCENCY: A FIRST TRIMESTER PRESENTATION OF FAMILIAL 13p SATELLITE DELETION
}

\author{
Uzun $\mathrm{I}^{1}$, Has $\mathrm{R}^{2}$, Alici E ${ }^{2}$, Ozdemir $\mathrm{M}^{3}$, İnan $\mathrm{C}^{4}$, Erzincan $\mathrm{S}^{2}$
}

*Corresponding Author: Dr. Isil Uzun, Department of Obstetrics and Gynecology, Acibadem Hospital, Halit Ziya Usakhıil Street, Bakırkoy, İstanbul, Turkey. Tel: +90-212-414-4400. Fax: +90-212-414-4152. E-mail: isiluzu@gmail.com

\begin{abstract}
Chromosome 13 is one of the acrocentric chromosomes of the human karyotype. Acrocentric chromosomes are the most variable chromosomes in the human karyotype and these variations appear to have no clinical consequences. To the best of our knowledge, this is the first reported case of a first trimester presentation of a 13 short arm satellite deletion with markedly increased nuchal translucency (NT). In this case, the 13p short arm satellite deletion was associated with increased NT in two pregnancies from the same couple.
\end{abstract}

Keywords: Chromosome 13; Nuchal translucency (NT); Pregnancy

\section{CASE REPORT}

A 35-year-old gravida 2, para 0 (G2P2A0) woman at 7-weeks gestation was admitted to our clinic for a routine prenatal check-up. Trans vaginal ultrasound revealed a pregnancy at 7 weeks of gestation.

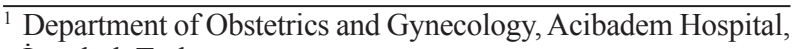
İstanbul, Turkey

${ }^{2}$ Department of Obstetrics and Gynecology, İstanbul University Medical School, İstanbul, Turkey

${ }^{3}$ Department of Obstetrics and Gynecology, Suleymaniye Maternity Hospital, İstanbul, Turkey

${ }^{4}$ Department of Obstetrics and Gynecology, Kulu Hospital, Konya, Turkey
}

Amniocentesis had been performed for increased nuchal translucency (NT) $(3.7 \mathrm{~mm})$ in her first pregnancy 8 years ago and short arm satellite deletion of chromosome 13 (13p) had been found in the chromosomal analysis. Consequently, parental karyotyping was performed and polymorphisms in chromosome 13 were also found (46XX13p- and 46XY13ps-). The parents were not related in any way. Medical history of the parents was uneventful. Intellectual and social development was completely normal and they had no history of psychiatric disorder. The intellectual, social, and physiological development of the first sibling of the family was also uneventful.

At the $12^{\text {th }}$ week of pregnancy, increased NT was detected during trans abdominal ultrasound examination. Nuchal translucency diameter was between 4.5 and $4.7 \mathrm{~mm}$. Chorion villus sampling was also performed at the $12^{\text {th }}$ week of pregnancy. There were no numerical aberrations. Microscopic evaluation of metaphase plates revealed a short arm satellite deletion of chromosome 13 (Figure 1). At the $20^{\text {th }}$ week of pregnancy, a detailed sonogram of the fetus was performed, which revealed no structural malformations. Subsequent prenatal examinations were uneventful. A planned Cesarean section was performed at the $39^{\text {th }}$ week of pregnancy and a healthy baby boy, weighing $3.7 \mathrm{~kg}$ and with a length of $51 \mathrm{~cm}$, was delivered with an Apgar score of between 9 and 10. The newborn was completely normal upon examination. Clinical examination of the baby at 2 years of age revealed a healthy baby with normal motor and mental development. 


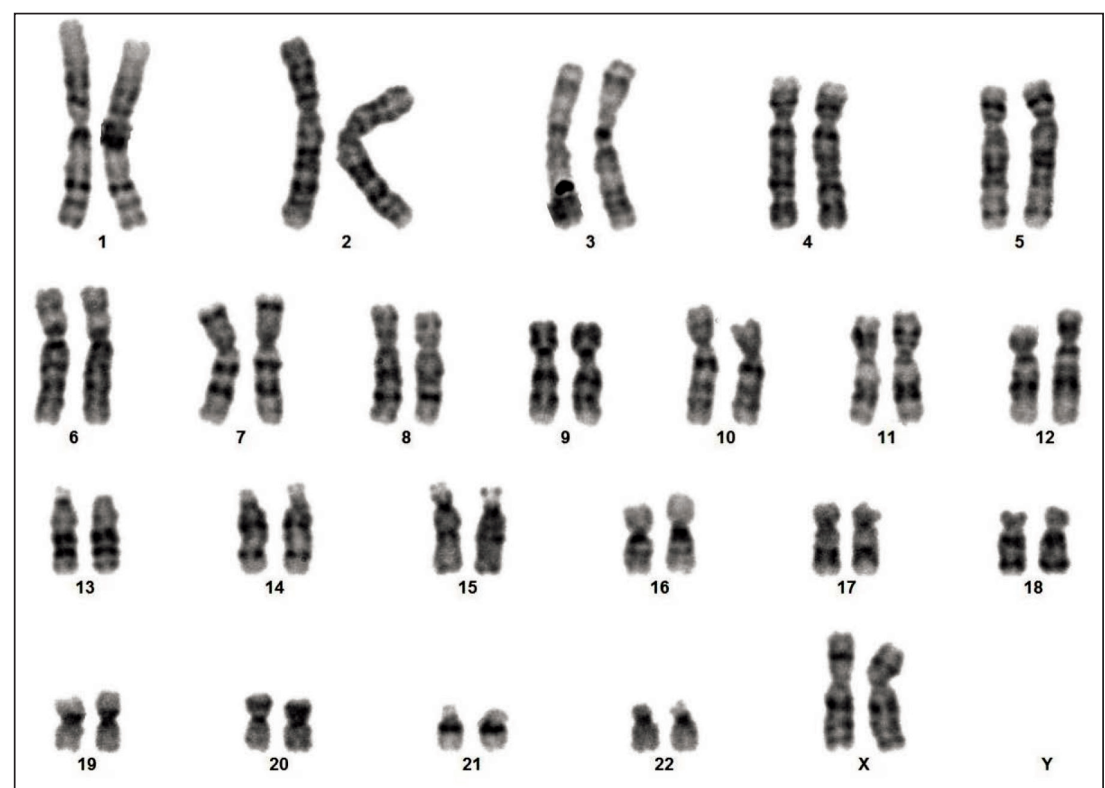

Figure 1. The 13p satellite deletion.

\section{DISCUSSION}

Chromosome 13 is one of the acrocentric chromosomes of the human karyotype, which also include chromosomes 14, 15, 21, and 22. The acrocentric chromosomes are the most variable chromosomes in the human karyotype. There is considerable shuffling of various repetitive DNA sequences consisting of the pericentromeric, short arm and satellite regions of these chromosomes. Variations include staining and/or size of the pericentromeric regions, short arms and satellites, as well as variation in the number and/or size of nucleolar organizing regions. None of these variations appear to have any clinical consequences [1].

Nielsen et al. [2] reported two cases with $13 p$ satellite deletions in their population study. The first case was totally normal; however, deviant behavior and criminality were observed in the second case. Studies indicate that the frequency of short arm satellites in acrocentric chromosomes in the population is between 0.5 and 1.0 per 1000 persons and that the lack of short arm satellite material does not have any deleterious effects on the phenotype [2]. In our case, $13 p$ satellite deletions were detected in both maternal and paternal karyotypes. The couple was not related to each other in any way.
To the best of our knowledge, this is the first report of a first trimester presentation of a 13p satellite deletion. The deletion is thought to be associated with enlarged NT; however, a congenital heart defect or other structural abnormalities known to cause nuchal edema was absent.

Increased NT is defined as an important marker for fetal chromosomal abnormalities and is used as a routine first-trimester screening test in many countries $[3,4]$. Some of the cases with increased NT are linked to submicroscopic chromosomal abnormalities that are typically missed by conventional karyotyping. Approximately $12.5 \%$ of fetuses with increased NT and an apparently normal karyotype have submicroscopic chromosomal abnormalities that are likely to be pathological [5].

The risk of genetic syndromes or neurodevelopmental delays in fetuses with increased NT at the first trimester and with normal karyotype is not yet well established. A systematic review demonstrated a $4.0 \%$ [ $95 \%$ confidence interval $(95 \% \mathrm{CI})(2.0-7.0)$ incremental yield for isolated NT. The most common pathogenic copy number variations reported were 22q11.2 deletion, 22q11.2 duplication, 10q26. $12 \mathrm{q} 26.3$ deletion and $12 \mathrm{q} 21 \mathrm{q} 22$ deletion [6]. In this report, increased NT in both cases are probably related to $13 \mathrm{p}$ presentation. Perinatologists should 
consider $13 p$ satellite deletions in their differential diagnoses in the event of an enlarged NT when this appears to be an isolated finding.

Short arm satellites in acrocentric chromosomes as $13 p$ satellite deletions may be a cause of increased NT. It should be noted that this is a relatively frequent phenomenon. This case demonstrates the importance of genetic counseling, karyotyping and microarray testing in the case of markedly increased NT. Non invasive prenatal testing via cell-free DNA is not adequate for increased NT. Correct diagnosis will help in the management of the present pregnancy and in counseling for future pregnancies.

Declaration of Interest. The authors report no conflicts of interest. The authors alone are responsible for the content and writing of this article.

\section{REFERENCES}

1. Wyandt HE. Human Chromosome Variation: Het-eromorphism and Polymorphism. New York, NY, USA: Springer, 2011.

2. Nielsen J, Friederich U, Hreidarson AB. Frequency of deletion of short arm satellites in acrocentric chromosomes. J Med Genet. 1974; 11(2): 177-180.
3. Snijders RJ, Noble P, Sebire N, Souka A, Nicolaides KH. UK Multicentre project on assessment of risk of trisomy 21 by maternal age and fetal nuchal-translucency thickness at 10-14 weeks of gestation. Fetal Medicine Foundation First Trimester Screening Group. Lancet. 1998; 352(9125): 343-346.

4. Leung TY, Chan LW, Law LW, Sahota DS, Fung TY, Lee TN, et al. First trimester combined screening for trisomy 21 in Hong Kong: Outcome of the first. J Matern Fetal Neonatal Med. 2009; 22(4): 300-304.

5. Leung TY, Vogel I, Lau TK, Chong W, Hyett JA, Peterson O. Identification of submicroscopic chromosomal aberrations in fetuses with increased nuchal translucency and apparently normal karyotype. Ultrasound Obstet Gynecol. 2011; 38(3): 314-319.

6. Grande M, Jansen FA, Blumenfeld YJ, Fisher A, Odibo AO, Haak MC, et al. Genomic microarray in fetuses with increased nuchal translucency and normal karyotype - A systematic review and meta-analysis. Ultrasound Obstet Gynecol. 2015; 46(6): 650-658. 
